Int. J. Odontostomat.,

15(4):1005-1008, 2021.

\title{
Desafíos de la Odontología Chilena en el Contexto del Nuevo Pacto Social
}

\author{
Challenges of Chilean Dental Profession in the Context of a New Social Contract
}

\author{
Valentina Fajreldin Chuaqui ${ }^{1,2}$; Marjorie Borgeat Meza ${ }^{3,4}$; Karen Danke Hausdorf4; \\ Bárbara Valenzuela Faunes ${ }^{5}$ \& Camila Torres Ceballos $^{5}$
}

\begin{abstract}
FAJRELDIN, C. V.; BORGEAT, M. M.; DANKE, H. K.; VALENZUELA, F. B. \& TORRES, C. C. Desafíos de la odontología chilena en el contexto del nuevo pacto social. Int. J. Odontostomat., 15(4):1005-1008, 2021.

RESUMEN: La actual crisis política y social derivada en un proceso constituyente, ha despertado el interés hacia importantes discusiones de las cuáles la profesión odontológica no es ajena. La salud orales un claro marcador de desventaja social, reflejando las inequidades en salud en todo su esplendor. La falta de aseguramiento de la salud bucal, sobre todo para la población adulta, lleva a la postergación de necesidades con el consecuente deterioro a la salud que esto implica. Por otro lado, la sobreoferta de odontólogos no viene a solucionar el problema de acceso, bajo un panorama de completa desregulación; el desempleo y la precariedad laboral es cada vez común entre profesionales jóvenes, quienes deben aceptar las condiciones de sus empleadores cuyas metas económicas incentivan la intervención por sobre la prevención, aumentando el riesgo de sobretratamiento y iatrogenia. El actual proceso constituyente representa una oportunidad para repensar el rol formador de las universidades y el rol social de la profesión ante el agudo escenario de inequidades en salud oral.
\end{abstract}

PALABRAS CLAVE: odontología, salud oral,políticas públicas, determinantes sociales de la salud, dentistas.

\section{INTRODUCCIÓN}

Chile se encuentra en una crisis política y social sin precedentes desde los años 70 , cuyo mayor síntoma es el estallido iniciado abruptamente en octubre de 2019, que derivó en un proceso constituyente con varios hitos y tareas en desarrollo; y refleja las profundas necesidades que motivan el deseo de cambio del pacto social tácito en el que Chile ha vivido desde la dictadura militar de 1973. El modelo neoliberal implementado en dictadura convirtió a Chile en uno de los países con mayor desigualdad socioeconómica a nivel mundial. Algunas de sus manifestaciones son la concentración del capital en el $1 \%$ más rico, bajos salarios con brecha de género, marcada inestabilidad e informalidad laboral (Cociña, 2017; Durán \& Kremerman, 2019; Instituto Nacional de Estadísticas, 2018). Se suma el rol in- suficiente del Estado respecto de las tareas de redistribución y provisión de seguridades para los ciudadanos ya que educación, salud y vivienda son servicios segmentados por clases, a lo que se suma la ausencia de un sistema de seguridad social efectivo en materia de pensiones (Cociña; Durán \& Kremerman). La salud es concebida constitucionalmente como un bien de consumo desde 1980; siendo la misma concebida fundamentalmente en su dimensión de atención y donde el Estado es garante de la libertad individual de elegir entre lo público y lo privado.

La actual discusión en torno del proceso constituyente nos abre la posibilidad de reflexionar sobre la odontología como disciplina y campo de saber y ha-

\footnotetext{
${ }^{1}$ Doctora en Antropología y Comunicación, Universidad Rovira i Virgili, España.

${ }^{2}$ Instituto de Investigación en Ciencias Odontológicas, Facultad de Odontología, Universidad de Chile, Santiago, Chile.

${ }^{3}$ Centro Interdisciplinario de Estudios en Salud, Facultad de Odontología, Universidad de Valparaíso, Valparaíso, Chile.

${ }^{4}$ Doctoranda en Ciencias Odontológicas, Facultad de Odontología, Universidad de Chile, Santiago, Chile.

${ }^{5}$ Estudiante Magíster en Ciencias Odontológicas, Facultad de Odontología, Universidad de Chile, Santiago, Chile.
} 
cer. A nivel mundial, a pesar de los grandes avances tecnológicos y mayor comprensión de la etiopatogenia de las enfermedades orales, éstas continúan siendo la condición de salud más prevalente en todas las edades (GBD 2017 Oral Disorders Collaborators et al., 2020). En Chile también existe una alta carga de morbilidad oral, con una prevalencia de caries cavitadas en ambos maxilares en población de $15 \mathrm{y}$ más años de $54,6 \%$, mientras que la prevalencia de dentición no funcional, es decir menos de 20 dientes en boca, es del $45,2 \%$ en personas de 45 a 64 años y de $81,7 \%$ en mayores de 65 años (Ministerio de Salud, 2017). En niños la prevalencia de historia de caries es de $70 \%$ a los 6 años (Ministerio de Salud, 2007) y de $62,6 \%$ a los 12 años (Soto et al., 2007).

El actual enfoque curativo-individual no es suficiente para controlar las enfermedades orales y lograr que todos puedan gozar de una dentición funcional de por vida (Baelum, 2011; Montenegro Martínez, 2011). La profesión requiere de cambios radicales con urgencia, que exigen repensar sus premisas y objetivos, ya sea en cuanto a la formación de profesionales, la provisión de servicios y la distribución de los equipos de salud oral (Fejerskov et al., 2013; Watt et al., 2019; Antunes et al., 2020). Otro aspecto relevante es comprender que los factores de riesgo conocidos de las enfermedades crónicas, entre ellas las orales y su distribución entre los distintos grupos sociales, no son necesariamente resultado de decisiones personales, sino que consecuencia de determinantes sociales que corresponden a factores estructurales que actúan a través de políticas y programas sociales deficientes, acuerdos económicos injustos y una mala gestión política (Organización Panamericana de la Salud, 2018). En Chile se observa en efecto una clara gradiente social en el estado de salud oral con mayor carga de enfermedad en grupos de menor nivel socioeconómico (Palomer Roggerone, 2016).

El Sistema de Salud del país es mixto tanto en el aseguramiento como en el financiamiento y la prestación de servicios (Arteaga, 2008). Esta fragmentación sistémica neoliberal ha creado una brecha de equidad entre los sectores público y privado (Núñez et al., 2020). El Fondo Nacional de Salud (FONASA) corresponde al asegurador público solidario para el $78 \%$ de la población. Las aseguradoras privadas (ISAPRE) cubren en tanto al $18 \%$ de la población y el $4 \%$ restante pertenece al seguro de las Fuerzas Armadas (Arteaga; Castillo-Laborde \& Villalobos Dintrans, 2013; Díaz, 2017). Mientras los afiliados a ISAPRE son personas de mayores ingresos, más jó- venes y con menos carga de enfermedad, los afiliados a FONASA presentan mayor vulnerabilidad socioeconómica, edad y carga de enfermedad. La red de atención estatal de salud acoge a los afiliados a FONASA, quienes también pueden acceder a prestadores privados a través de la "modalidad libre elección" mediante copago. Los afiliados a ISAPRE reciben atención de salud en prestadores privados con cobertura variable según el plan de salud (CastilloLaborde \& Villalobos Dintrans; Díaz).

La odontología en Chile también se ha dividido entre el ejercicio público y privado de la profesión. El país cuenta a diciembre del 2019 con un total de 25.440 odontólogos registrados (Superintendencia de Salud, 2020), lo que corresponde a una proporción de un dentista cada 751 habitantes, una de las tasas más altas del mundo (Gallagher \& Hutchinson, 2018), y continúan egresando anualmente más de 1.500 nuevos profesionales (Arellano-Villalón \& Fuentes, 2020). Se estima que el $76 \%$ se desempeña exclusivamente en el sector privado (Ministerio de Salud, 2018). Sobre el ejercicio en el ámbito público, hasta hace un par de décadas, la oferta del sector se limitaba a las atenciones de urgencia y con tratamientos en un porcentaje de cobertura muy bajo; habiéndose incrementado gradualmente de la mano de un aumento de los profesionales disponibles y el desarrollo de programas de salud oral que priorizan a la población infanto-juvenil.

La reforma de salud del 2005, incorporó un programa de garantías explícitas en salud (GES) que aseguran el acceso, calidad, oportunidad y protección financiera para toda la población. Éstas incluyen:Fisura labio palatina, Salud integral para niños y niñas de 6 años, Urgencias odontológicas ambulatoria, Atención integral adulto de 60 años y Salud integral de la embarazada (Jara et al., 2018). Existen otros programas a nivel de Atención Primaria que entregan atención odontológica a niños, niñas y adolescentes con un enfoque fundamentalmente preventivo. Con respecto a la población adulta, existen programas con cupos y prestaciones limitadas como el "Más Sonrisas para Chile", "Hombres de Escasos Recursos" y el programa de "Mejoramiento del Acceso a la Atención de Morbilidad Odontológica de los Adultos", que poseen principalmente un enfoque curativo (Cartes-Velásquez, 2020), que trata de contener el alto nivel de daño oralde la población. La atención odontológica del adulto en el sector público (a partir de los 20 años) no sigue la lógica de mantener a la población bajo control odontológico y prevenir activamente el desarrollo de enfermedades orales. Por ello existe una desconexión 
en la manera en que se pretende mejorar la salud oral de toda la población, siendo insuficiente la forma en que se han dispuesto los programas orientados a mejorar la salud oral de la población adulta, variando su disponibilidad de acuerdo con criterios de oferta de especialistas y de tipo administrativo, existiendo una larga lista de espera (Fundación Sonrisas, 2018). A nivel de atención secundaria y terciaria, el país cuenta con especialidades odontológicas disponibles en los Servicios de Salud de acuerdo a criterios de oferta, donde 1 de cada 4 personas está a la espera de una especialidad odontológica, siendo el promedio de la espera de 511 días (17 meses) (Fundación Sonrisas).

Por otra parte, la atención odontológica en el sector privado se ha expandido con la emergencia de los llamados mega prestadores. A diferencia del sector público y salvo las GES odontológicas, no existen programas de salud oraldefinidos y se concentra el mayor número de especialistas; el modelo incentiva lo curativo, aumentando los riesgos de sobretratamiento y iatrogenia con consecuencias preocupantes para la salud oral de la población, sin lograr resolver las dificultades de acceso para la gran mayoría de la población adulta sincapacidad de pago (Holden, 2018; Cartes-Velásquez). Visto desde la experiencia laboral, los odontólogos deben aceptar condiciones de trabajo sin contrato laboral, sin seguridad social, pago por porcentaje y acciones efectuadas y con metas de rentabilidad de los empleadores.

Reconociendo los avances que la odontología ha tenido en el país, ante un escenario de desigualdades sociales, un sistema de salud segmentado, aumento indiscriminado de escuelas de odontología y precariedad laboral, ¿cuál debe ser la dirección que tome la odontología en contexto? (Abadía-Barrero, 2006). Pese a la discusión parece ser que el gremio odontológico no ha levantado con suficiente fuerza la voz de alerta sobre la necesidad de cambiar el enfoque individual y rehabilitador de la atención odontológica adulta, de regular la formación de los odontólogos -número, perfil y competencias; coherente con un paradigma de promoción y conservación de la salud oral y bienestar de la población.

Coincidiendo con que la profesión odontológica es un conjunto de prácticas y de actores (Abadía-Barrero), a la discusión le hacen falta además mayor participación de las universidades tanto en su rol formador como en su papel de aporte a la investigación hacia las políticas públicas orientadas a lograr el mejor estándar de atención odontológica con un enfo- que preventivo y promotor (Fejerskov, 2017). Múltiples factores como la falta de acuerdo en la definición de los problemas de la profesión y sus causas, escasez de indicadores que muestren y monitoreen la severidad del problema, ausencia de propuestas concretas y sustentadas en evidencia científica para abordar los desafíos, instituciones y organizaciones carentes de liderazgo y de un diálogo coherente con el Estado, entre otros, dificultan el posicionamiento de este tema como prioridad política (Benzian et al., 2011). El contexto en el que se encuentra Chile, representa una ventana de oportunidad política para corregir el rumbo de la profesión, instalar la salud oral como derecho, además de poder discutir el rol social que debe cumplir la profesión en nuestros días

FAJRELDIN, C. V.; BORGEAT, M. M.; DANKE, H. K.; VALENZUELA, F. B. \& TORRES, C. C. Challenges of the Chilean dental profession in the context of a new social contract. Int. J. Odontostomat., 15(4):1005-1008, 2021.

ABSTRACT: The current political and social crisis which derived in a constituent process, has aroused interest in important discussions that also include the dental profession. Oral health is a clear marker of social disadvantage, reflecting health inequities in every aspect. The lack of public oral health policies, particularly with regard to the adult population, whose needs when postponed, suffer the consequent deterioration of their oral health. On the other hand, the oversupply of dentists does not solve the problem in a scenario of total deregulation. Unemployment and job insecurity are increasingly common among young professionals, who must accept conditions imposed by their employers, whose financial objectives often encourage intervention rather than prevention, thereby increasing the risk of overtreatment and iatrogenesis. The current process represents an opportunity to reevaluate the educational role of universities, and the social role of dentists in a scenario with serious oral health inequities.

KEY WORDS: dentistry, oral health, public policy, social determinants of health, dentists.

\section{REFERENCIAS BIBLIOGRÁFICAS}

Abadía Barrero, C. E. Pobreza y desigualdades sociales: un debate obligatorio en salud oral. Acta Bioeth., 12(1):9-22, 2006.

Antunes, J. L. F.; da Cunha, A. R. \& Petti, S. Rethinking dentistry and dental teaching. Oral Dis., 26(1):6-11, 2020.

Arellano-Villalón, M. \& Fuentes, R. From admission to professional practice, a review of dental programs in Chile. Int. J. Odontostomat., 14(4):623-31, 2020.

Arteaga, O. Desde el Diseño a la Implementación de Políticas Públicas: el Caso de la Reforma de Salud en Chile. Transformaciones Sociales y Sistemas de Salud en América Latina. FLACSO Quito, Serie 50, 2008. pp.121-2. 
Baelum, V. Dentistry and population approaches for preventing dental diseases. J. Dent., 39:S9-S19, 2011.

Benzian, H.; Hobdell, M.; Holmgren, C.; Yee, R.; Monse, B.; Barnard, J. T. \& van Palenstein Helderman, W. Political priority of global oral health: an analysis of reasons for international neglect. Int. Dent. J., 61(3):124-30, 2011.

Cartes-Velásquez, R. Salud bucal en Chile, situación actual y desafíos futuros. Odontol. Sanmarquina, 23(2):189-96, 2020.

Castillo-Laborde, C. \& Villalobos Dintrans, P. Caracterización del gasto de bolsillo en salud en Chile: una mirada a dos sistemas de protección. Rev. Med. Chile, 141(11):1456-63, 2013.

Cociña, M. Desiguales: Orígenes, Cambios y Desafíos de la Brecha Social en Chile. Ginebra, Programa de las Naciones Unidas para el Desarrollo (PNUD), 2017.

Díaz, Á. Mercado de las Isapres en Chile. Rev. Chil. Salud Pública, 21(1):91-6, 2017.

Durán, G. \& Kremerman, M. Los bajos salarios de Chile. Un análisis de la encuesta CASEN. Ideas para el Buen Vivir (14), 2019. Disponible en: https://www.fundacionsol.cl/ cl_luzit_herramientas/static/wp-content/uploads/2019/04/Salarios-al-Li \%CC \%81mite-2017-NV2-1.pdf

Fejerskov, O. The Dental Profession in the 21st Century: Proud Past, Questionable Present, Challenging Future. The Future of Oral Health, 2017. Disponible en: https:// lacascada.pressbooks.com/chapter/chapter-1/

Fejerskov, O.; Escobar, G.; Jøssing, M. \& Baelum, V. A functional natural dentition for all-and for life? The oral healthcare system needs revision. J. Oral Rehabil., 40(9):707-22, 2013.

Fundación Sonrisas. ¿Cuánto Esperan Las Sonrisas de Chile? Análisis Lista Espera Odontológica. Santiago de Chile, Fundación Sonrisas, 2018. Disponible en: http:// fundacionsonrisas.cl/wp-content/uploads/2018/09/estudiofundacion-sonrisas-mier19SEP.pdf

Gallagher, J. E. \& Hutchinson, L. Analysis of human resources for oral health globally: inequitable distribution. Int. Dent. J., 68(3):183-9, 2018.

GBD 2017 Oral Disorders Collaborators; Bernabe, E.; Marcenes, W.; Hernandez, C.; Bailey, J.; Abreu, L.; Alipour, V.; Amini, S.; Arabloo, J.; Arefi, Z.; et al. Global, regional, and national levels and trends in burden of oral conditions from 1990 to 2017: a systematic analysis for the global burden of disease 2017 study. J. Dent. Res., 99(4):362-73, 2020.

Holden, A. C. L. Consumer-driven and commercialised practice in dentistry: an ethical and professional problem? Med. Health Care Philos., 21(4):583-9, 2018.

Instituto Nacional de Estadísticas. Síntesis de resultados Censo 2017. Santiago de Chile, Gobierno de Chile, 2018. Disponible en: https://www.censo2017.cl/descargas/home/sintesisde-resultados-censo2017.pdf

Jara, G.; Werlinger, F. \& Cabello, R. Sinopsis de la Situación de Salud Oral en Chile - Parte I: Garantías Explícitas y Guías Clínicas. Rev. Clín. periodoncia Implantol. Rehabil.,11(3):18790, 2018.

Ministerio de Salud. Diagnóstico Nacional de Salud Bucal en los Niños de 6 Años. Santiago de Chile, Ministerio de Salud, Gobierno de Chile, 2007.

Ministerio de Salud. Informe Sobre Brechas Del Personal de SaIud en los Servicios de Salud y Estado de Situación de los Recursos Humanos de Salud en Chile. Santiago de Chile, Ministerio de Salud, Gobierno de Chile, 2018.

Ministerio de Salud. Subsecretaría de Salud Pública. Encuesta Nacional de Salud 2016-2017 Primeros Resultados. Santiago de Chile, Ministerio de Salud, Gobierno de Chile, 2017.

Montenegro Martínez, G. Un nuevo enfoque de la salud oral: una mirada desde la salud pública. Univ. Odontol., 30(64), 2011.
Núñez, A.; Manzano, C. \& Chi, C. Health outcomes, utilization, and equity in Chile: an evolution from 1990 to 2015 and the effects of the last health reform. Public Health, 178:38-48, 2020.

Organización Panamericana de la Salud. Sociedades Justas: Equidad en la Salud y Vida Digna. Resumen Ejecutivo del Informe de la Comisión de la Organización Panamericana de la Salud sobre Equidad y Desigualdades en Salud en las Américas. Washington D.C., Organización Panamericana de la Salud, 2018.

Palomer Roggerone, L. Inequidades en salud bucal: Factores que determinan su realidad en Chile. Acta Bioeth., 22(2):3159, 2016.

Soto, L.; Tapia, R.; Jara, G.; Rodríguez, G.; Urbina, T. \& Venegas, C. Diagnóstico Nacional de Salud Bucal del Adolescente de 12 Años y Evaluación del Grado de Cumplimiento de los Objetivos Sanitarios de Salud Bucal 2000-2010. Santiago de Chile, Facultad de Odontología, Universidad Mayor, 2007.

Superintendencia de Salud. Registro nacional de prestadores individuales de salud. (obtenido a través de la ley de transparencia 20.285. Respuesta a su solicitud de información AO006T0003258, 27/01/2020). Santiago de Chile, Superintendencia de Salud, 2020.

Watt, R. G.; Daly, B.; Allison, P.; Macpherson, L. M.; Venturelli, R.; Listl, S.; Weyant, R. J.; Mathur, M. R.; Guarnizo-Herreño, C. C.; Celeste, R. K.; et al. Ending the neglect of global oral health: time for radical action. Lancet, 394(10194):261-72, 2019.

Dirección de correspondencia:

Karen Danke Hausdorf

Universidad de Chile

Santiago

CHILE

E-mail: kdankeh@odontologia.uchile.cl 